Article

\title{
First Integrals of Two-Dimensional Dynamical Systems via Complex Lagrangian Approach
}

\author{
Muhammad Umar Farooq ${ }^{1}$, Chaudry Masood Khalique ${ }^{2}$ and Fazal M. Mahomed ${ }^{3, *}$ \\ 1 Department of Basic Sciences \& Humanities, College of E \& ME, National University of Sciences and \\ Technology, H-12, Islamabad 44000, Pakistan; m_ufarooq@yahoo.com \\ 2 International Institute for Symmetry Analysis and Mathematical Modelling, Department of Mathematical \\ Sciences, North-West University, Mafikeng Campus, Private Bag X 2046, Mmabatho 2735, South Africa; \\ Masood.Khalique@nwu.ac.za \\ 3 DST-NRF Centre of Excellence in Mathematical and Statistical Sciences, School of Computer Science and \\ Applied Mathematics, University of the Witwatersrand, Wits, Johannesburg 2050, South Africa \\ * Correspondence: Fazal.Mahomed@wits.ac.za
}

Received: 22 July 2019; Accepted: 10 September 2019; Published: 4 October 2019

\begin{abstract}
The aim of the present work is to classify the Noether-like operators of two-dimensional physical systems whose dynamics is governed by a pair of Lane-Emden equations. Considering first-order Lagrangians for these systems, we construct corresponding first integrals. It is seen that for a number of forms of arbitrary functions appearing in the set of equations, the Noether-like operators also fulfill the classical Noether symmetry condition for the pairs of real Lagrangians and the generated first integrals are reminiscent of those we obtain from the complex Lagrangian approach. We also investigate the cases in which the underlying systems are reducible via quadrature. We derive some interesting results about the nonlinear systems under consideration and also find that the algebra of Noether-like operators is Abelian in a few cases.
\end{abstract}

Keywords: complex Lagrangian approach; coupled Lane-Emden systems; Noether-like operators; first integrals

\section{Introduction}

The famous generalized Lane-Emden (LE) equation

$$
\frac{d^{2} y}{d x^{2}}+\frac{n}{x} \frac{d y}{d x}+F(y)=0,
$$

where $n$ is a real constant and $F(y)$ is a continuous function of $y$, has been used to formulate many problems in mathematical physics and astrophysics. For instance, for $n=2$ and $F(y)=y^{r}$ (where $r=0,1 \& 5$ ) it has diverse applications in applied mathematics particularly in the field of stellar dynamics [1]. In the case $F(y)=\left(y^{2}-A\right)^{3 / 2}$, the above equation was used to study the gravitational potential of degenerate white dwarf star [1]. Moreover, for other forms of $F(y)=e^{y}, e^{-y}$, Equation (1) describes isothermal gas spheres and in the theory of thermionic currents respectively [2-6]. Therefore, in having intriguing mathematical and physical properties, Equation (1) has been investigated from various other aspects as well. For example, a number of techniques have been employed which include power series, differential transformation, numerical, perturbation and Adomian decomposition to seek solutions of the above equation [7-9]. A pretty comprehensive review on this equation by Wang is also available who mentioned more than 140 references related to it [10]. In the recent past, the authors [11] considered the above equation in variational form and classify it with respect to Noether symmetries and presented the solution by constructing the corresponding 
first integrals. Furthermore, some other related work on Lie symmetries towards finding solution of LE-type equations can also be seen in [12].

A natural two-dimensional extension of (1) can be expressed as

$$
\begin{aligned}
& \frac{d^{2} f}{d x^{2}}+\frac{n}{x} f^{\prime}+P(x) F(f, g)=0, \\
& \frac{d^{2} g}{d x^{2}}+\frac{n}{x} g^{\prime}+P(x) G(f, g)=0,
\end{aligned}
$$

in which the functions $F$ and $G$ provide couplings in the above system. This pair of LE-equations appears in various practical situations, for example, its use in the modeling of chemical reaction, population evolution and pattern formation, etc. [13]. Due to its widespread applications, the above system has been studied by many authors. For specific forms of $P(x), F(f, g), G(f, g)$ for example, $P(x)=1$ and $F(f, g)=F(g), G(f, g)=G(f)$, etc., a considerable amount of work has been done on establishing the existence and uniqueness results of LE-systems (2) as well as other relevant classes in [14-17]. Similarly, for the above stated forms of $P(x), F$ and $G$ along with few more, ample literature on the classification of the above system with respect to Lie symmetries, Noether symmetries and production of associated first integrals is available. In this regard, the interested reader is referred to [18-21] (and references in these papers for more details). Seeking the symmetry properties and conservation laws of LE-system (2) with respect to different cases which we present here is novel. It is essential as it generates further interest in researchers who can apply it to a more specific scenario as, e.g., in [18] for $P(x)=1$. Moreover, unless this more general approach is presented in the literature, the application of (2) in specific cases can likely be overlooked due to its intrinsic difficult nature. Although we are considering only two forms of the function $P(x)$ which are power laws and related to the index $n$ in (2) (the first in a general manner and the second as $-2 n$ ), there may be others in classifying LE-systems with respect to Noether symmetries and conservation laws. That is why the presentation of this new system of LE-equations is significant in terms of widening the possible scope of theoretical investigation as well as applications of (2).

The search of new conservation laws for systems of dynamical equations has important physical consequences. The main objective of the present work is to perform Noether-like operator classification of the generalized version of LE-system (2) with respect to first-order Lagrangians. We take $P(x)$ as a non-constant power law function and $F(f, g), G(f, g)$ in their most general forms and derive conservation laws of the ensuing systems. To the best of our knowledge, this investigation of group theoretic properties of (2) has not been performed as yet. We apply the complex Lagrangian formalism which has been recognized as a legitimate technique to perform this classification of a class of systems of LE-equations.

The outline of the paper is as follows: The next section constitutes some basic expressions which we use in the sequel. In Section 3, we classify (2) with respect to Noether-like operators and find related first integrals. The cases in which the Noether-like operators become Noether symmetries are also discussed in detail. Finally, Section 4 concludes the discussion.

\section{Basic Definitions and Expressions for Noether-Like Operators and Conservation Laws}

The determination of symmetries of a coupled system of nonlinear ordinary differential equations (ODEs) which leave the action integral, up to a gauge function, invariant is a nontrivial task. The authors [22-24] have introduced a complex Lagrangian approach to uncover the relationship between symmetries and first integrals of coupled systems of nonlinear equations. As we proceed we see how this new technique helps in reducing the complexity of the problem and offers a great deal of information about the inverse problem, algebraic properties and first integrals of the underlying systems of LE-equations. Here, we describe some salient features of Noether-like operators and first integrals of two Euler-Lagrange equations which are taken from $[23,24]$ and will be used in the forthcoming section. 
Suppose we have a physical system whose dynamics is governed by a pair of coupled nonlinear ODEs

$$
\begin{aligned}
& f^{\prime \prime}=F\left(x, f, g, f^{\prime}, g^{\prime}\right), \\
& g^{\prime \prime}=G\left(x, f, g, f^{\prime}, g^{\prime}\right) .
\end{aligned}
$$

We assume that the above system (3) has a complex structure and can be mapped to a base equation $u^{\prime \prime}=W\left(x, u, u^{\prime}\right)$ via transformations $u=f+i g$ and $W=F+i G$ (see for example [22-24], for more details). Following this approach a number of studies have been done in finding the symmetries, linearization, reduction of order and conservation laws for a wide class of systems of two nonlinear equations as shown in (3) [25-28]. We assume that the system (3) possesses a variational form, i.e., there exists a pair of first-order Lagrangians $L_{1}\left(x, f, g, f^{\prime}, g^{\prime}\right)$ and $L_{2}\left(x, f, g, f^{\prime}, g^{\prime}\right)$ such that $L=L_{1}+i L_{2}$ and which can be retrieved from the pair of Euler-Lagrange-type equations

$$
\begin{aligned}
& \frac{\partial L_{1}}{\partial f}+\frac{\partial L_{2}}{\partial g}-\frac{d}{d x}\left(\frac{\partial L_{1}}{\partial f^{\prime}}+\frac{\partial L_{2}}{\partial g^{\prime}}\right)=0, \\
& \frac{\partial L_{2}}{\partial f}-\frac{\partial L_{1}}{\partial g}-\frac{d}{d x}\left(\frac{\partial L_{2}}{\partial f^{\prime}}-\frac{\partial L_{1}}{\partial g^{\prime}}\right)=0 .
\end{aligned}
$$

\section{Noether-like symmetry conditions:}

The operators $\mathbf{X}_{1}$ and $\mathbf{X}_{2}$ given by

$$
\mathbf{x}_{1}=\xi_{1}(x, f, g) \frac{\partial}{\partial x}+\eta_{1}(x, f, g) \frac{\partial}{\partial f}+\eta_{2}(x, f, g) \frac{\partial}{\partial g}
$$

and

$$
\mathbf{X}_{2}=\xi_{2}(x, f, g) \frac{\partial}{\partial x}+\eta_{2}(x, f, g) \frac{\partial}{\partial f}-\eta_{1}(x, f, g) \frac{\partial}{\partial g}
$$

are said to be Noether-like operators with respect to the Lagrangians $L_{1}$ and $L_{2}$ if the following conditions for appropriate gauge functions $A_{1}, A_{2}$ hold

$$
\begin{aligned}
& \mathbf{X}_{1}^{(1)} L_{1}-\mathbf{X}_{2}^{(1)} L_{2}+\left(D_{x} \xi_{1}\right) L_{1}-\left(D_{x} \xi_{2}\right) L_{2}=D_{x} A_{1} \\
& \mathbf{X}_{1}^{(1)} L_{2}-\mathbf{X}_{2}^{(1)} L_{1}+\left(D_{x} \xi_{1}\right) L_{2}+\left(D_{x} \xi_{2}\right) L_{1}=D_{x} A_{2},
\end{aligned}
$$

where $D_{x}$ denotes the total derivative with respect to $x$.

\section{Noether-like Theorem:}

If $L_{1}$ and $L_{2}$ are two real Lagrangians of system (3) and $\mathbf{X}_{1}$ and $\mathbf{X}_{2}$ are the associated Noether-like operators, then the following expressions

$$
\begin{aligned}
& I_{1}=\xi_{1} L_{1}-\xi_{2} L_{2}+\frac{\partial L_{1}}{\partial f^{\prime}}\left(\eta_{1}-f^{\prime} \xi_{1}-g^{\prime} \eta_{2}\right)-\frac{\partial L_{2}}{\partial f^{\prime}}\left(\eta_{2}-f^{\prime} \xi_{2}-g^{\prime} \xi_{1}\right)-A_{1}, \\
& I_{2}=\xi_{1} L_{2}+\xi_{2} L_{1}+\frac{\partial L_{2}}{\partial f^{\prime}}\left(\eta_{1}-f^{\prime} \xi_{1}-g^{\prime} \xi_{2}\right)+\frac{\partial L_{1}}{\partial f^{\prime}}\left(\eta_{2}-f^{\prime} \xi_{2}-g^{\prime} \xi_{1}\right)-A_{2} .
\end{aligned}
$$

provide two conserved quantities for the coupled Euler-Lagrange system (3). For its proof, one is referred to [24].

\section{Classification of Lane-Emden System w.r.t Noether-Like Operators and Corresponding First Integrals}

It is quite well-known that first integrals (constants of the motion) are of great importance in the analysis of behavior of the underlying systems. The celebrated Noether's theorem [29] provides a 
simple procedure for production of conserved quantities of Euler-Lagrange systems once the related Noether symmetry generators are available. We see that these operators not only provide conserved quantities but also reduce the equation via quadrature.

In this section we intend to perform a complete classification of the two-dimensional LE-system (2) admitting a variational form with respect to Noether-like operators and establish first integrals by choosing $P(x)$ as non-constant functions with arbitrary forms of $F$ and $G$. We point out here that this investigation has not been carried out before. We commence by assuming that the dynamical system represented by the LE-equations (2) admits the following pair of first-order Lagrangians

$$
\begin{aligned}
& L_{1}=\frac{1}{2} x^{n}\left(f^{\prime 2}-g^{\prime 2}\right)-x^{n} P(x) \int(F d f-G d g), \\
& L_{2}=x^{n}\left(f^{\prime} g^{\prime}\right)-x^{n} P(x) \int(G d f+F d g) .
\end{aligned}
$$

We find eight cases and present the results in a simple and straightforward manner. We observe that for many cases the Noether-like operators also fulfill the classical Noether symmetry condition and the constructed first integrals are the same as those we obtain by employing the complex Lagrangian approach. We provide two Cases A. and B.

$$
\text { A. } P(X)=X^{\alpha-1}
$$

Now by assuming this specific form of $P(x)$ and different values of $n$ and $\alpha$ in (2) and accordingly in (7), we proceed as:

Case 1. Herein $P(x)=1$. If we take $F(f, g)$ and $G(f, g)$ as linear functions of $f$ and $g$, we obtain a system of linear equations which can further be converted into the simplest two-dimensional system $f^{\prime \prime}=g^{\prime \prime}=0$. With these choices we find by employing (5) and (6), nine Noether-like operators and ten first integrals as shown in the following table.

\begin{tabular}{cc}
\hline Noether-Like Operators & First Integrals \\
\hline$\partial_{x}$ & $I_{1,1}=f^{\prime 2}-g^{\prime 2}$ \\
$I_{1,2}=f^{\prime} g^{\prime}$ \\
\hline$\partial_{f}$, & $I_{2,1}=f^{\prime}$ \\
$\partial_{g}$ & $I_{2,2}=g^{\prime}$ \\
\hline$x \partial_{f}$, & $I_{3,1}=x f^{\prime}-f$ \\
$x \partial_{g}$ & $I_{3,2}=x g^{\prime}-g$ \\
\hline $2 x \partial_{x}+f \partial_{f}+g \partial_{g}$, & $I_{4,1}=-x\left(f^{\prime 2}-g^{\prime 2}\right)+f f^{\prime}-g g^{\prime}$ \\
$g \partial_{f}-f \partial_{g}$ & $I_{4,2}=-x f^{\prime} g^{\prime}+f g^{\prime}+f^{\prime} g$ \\
\hline$x^{2} \partial_{x}+x\left(f \partial_{f}+g \partial_{g}\right)$, & $I_{5,1}=x\left(f f^{\prime}-g g^{\prime}\right)-\frac{x^{2}}{2}\left(f^{\prime 2}-g^{\prime 2}\right)-\frac{1}{2}\left(f^{2}-g^{2}\right)$ \\
$x\left(g \partial_{f}-f \partial_{g}\right)$ & $I_{5,2}=x\left(f g^{\prime}+f^{\prime} g\right)-x^{2} f^{\prime} g^{\prime}-f g$ \\
\hline
\end{tabular}

For details the reader can see [24].

Case 2. Here $n$ and $\alpha$ are related as $n=\frac{1-\alpha}{2}$, and $F(f, g), G(f, g)$ are arbitrary. From Equation (5) with the aid of (7), we obtain $\xi_{1}=x^{\frac{1-\alpha}{2}}, \eta_{1}=\eta_{2}=0$ with $A_{1}, A_{2}$ as constants. Only one Noether-like operator exists, viz., $\mathbf{X}=x^{\frac{1-\alpha}{2}} \frac{\partial}{\partial x}$. Therefore, by utilizing this operator with the related Lagrangians in (6), we deduce the following first integrals.

$$
\begin{aligned}
& I_{1}=\frac{1}{2} x^{1-\alpha}\left(f^{\prime 2}-g^{\prime 2}\right)+\int(F d f-G d g), \\
& I_{2}=x^{1-\alpha}\left(f^{\prime} g^{\prime}\right)+\int(G d f+F d g) .
\end{aligned}
$$


It is worth seeing that the operator $\mathbf{X}$ also fulfills the classical Noether symmetry condition and the resulting Noetherian integrals are reminiscent of those given in (8).

Case 3. $F(f, g)=\frac{\alpha_{1}}{2} \ln \left(f^{2}+g^{2}\right)+\gamma f+\delta, \alpha_{1} \neq 0$ and $G(f, g)=\alpha_{1} \arctan (g / f)+\gamma g, \alpha_{1} \neq 0$. If $n=\frac{1-\alpha}{2}$, we obtain $\xi_{1}=x^{\frac{1-\alpha}{2}}, \xi_{2}=0$ and $\eta_{1}=\eta_{2}=0$ while $A_{1}=A_{2}=k, k$ a constant. This case is subsumed in Case 2.

Case 4. For this case, let us take $F=\left(f^{2}+g^{2}\right)^{r / 2} \cos \theta, G=\left(f^{2}+g^{2}\right)^{r / 2} \sin \theta$, where $\theta=\arctan (g / f)$. We discuss the following subcases.

Case 4.1 If $n=\frac{r+2 \alpha+1}{r-1}$, we determine from the Noether-like symmetry conditions (5) $\xi_{1}=x$, $\xi_{2}=0$ and $\eta_{1}=\frac{\alpha+1}{1-r} f, \eta_{2}=\frac{\alpha+1}{1-r} g$ while $A_{1}$ and $A_{2}$ appear as constants. Hence the Noether-like operators take the form

$$
\mathbf{X}_{1}=x \frac{\partial}{\partial x}+\left(\frac{1+\alpha}{1-r}\right) f \frac{\partial}{\partial f}+\left(\frac{1+\alpha}{1-r}\right) g \frac{\partial}{\partial g} \text { and } \mathbf{X}_{2}=\frac{1+\alpha}{1-r}\left(g \frac{\partial}{\partial f}-f \frac{\partial}{\partial g}\right)
$$

Now utilization of (6) with $\mathbf{X}_{1}, \mathbf{X}_{2}$ gives rise to the following two integrals for (2):

$$
\begin{aligned}
& I_{1}=\frac{1}{2} x^{\frac{2(\alpha+r)}{r-1}}\left(f^{\prime 2}-g^{\prime 2}\right)+\frac{\alpha_{1}}{r+1} x^{\frac{(r+1)(\alpha+1)}{r-1}}\left(f^{2}+g^{2}\right)^{\frac{r+1}{2}} \cos \theta-\frac{\alpha+1}{1-r} x^{\frac{r+2 \alpha+1}{r-1}}\left(f f^{\prime}-g g^{\prime}\right), \\
& I_{2}=x^{\frac{2(\alpha+r)}{r-1}} f^{\prime} g^{\prime}+\frac{\alpha_{1}}{r+1} x^{\frac{(r+1)(\alpha+1)}{r-1}}\left(f^{2}+g^{2}\right)^{\frac{r+1}{2}} \sin \theta-\frac{\alpha+1}{1-r} x^{\frac{r+2 \alpha+1}{r-1}}\left(f g^{\prime}+f^{\prime} g\right),
\end{aligned}
$$

Using the transformations $f=w_{1} x^{\frac{\alpha+1}{1-r}}$ and $g=w_{2} x^{\frac{\alpha+1}{1-r}}$, the pair of integrals (9) can be converted into integrable form as

$$
\int \frac{d w}{ \pm \sqrt{(\alpha+1)^{2}(1-r)^{-2} w^{2}-2 \alpha_{1}(1+r)^{-1} w^{1+r}-2 K_{1}}}=\ln x K_{2}
$$

where $K_{1}$ and $K_{2}$ are constants.

Case 4.2 If $n=\frac{r+\alpha+2}{r+1}$ with $r \neq-1$, Equation (5) provides $\xi_{1}=x^{\frac{r-\alpha}{r+1}}, \xi_{2}=0, \eta_{1}=-\frac{\alpha+1}{r+1} x^{-\frac{\alpha+1}{r+1}} f$, $\eta_{2}=-\frac{\alpha+1}{r+1} x^{-\frac{\alpha+1}{r+1}} g$ and the related gauge functions are $A_{1}=\frac{(\alpha+1)^{2}}{2(r+1)^{2}}\left(f^{2}-g^{2}\right)+C, A_{2}=\frac{(\alpha+1)^{2}}{(r+1)^{2}} f g$, where $C$ is constant. Now the Noether-like therorem (6) along with these operators and corresponding Lagrangians, reveal the first integrals

$$
\begin{aligned}
& I_{1}=\frac{1}{2} x^{2}\left(f^{\prime 2}-g^{\prime 2}\right)+\left(\frac{\alpha_{1}}{r+1}\right) x^{1+\alpha}\left(f^{2}+g^{2}\right)^{\frac{r+1}{2}} \cos \theta+\left(\frac{\alpha+1}{r+1}\right) x\left(f f^{\prime}-g g^{\prime}\right)+\frac{1}{2}\left(\frac{\alpha+1}{r+1}\right)^{2}\left(f^{2}-g^{2}\right), \\
& I_{2}=x^{2} f^{\prime} g^{\prime}+\left(\frac{\alpha_{1}}{r+1}\right) x^{1+\alpha}\left(f^{2}+g^{2}\right)^{\frac{r+1}{2}} \sin \theta+\left(\frac{\alpha+1}{r+1}\right) x\left(f g^{\prime}+f^{\prime} g\right)+\left(\frac{\alpha+1}{r+1}\right)^{2} f g,
\end{aligned}
$$

for the ensuing systems of LE-equations. Moreover, by applying the transformations $f=w_{1} x^{-\frac{\alpha+1}{r+1}}$ and $g=w_{2} x^{-\frac{\alpha+1}{r+1}}$, we can convert the pair of $I_{1}, I_{2}$ given in (11) in the form

$$
\int \frac{d w}{ \pm \sqrt{K_{1}-2 \alpha_{1}(r+1)^{-1} w^{r+1}}}=\left(\frac{r+1}{\alpha+1}\right) x^{\frac{\alpha+1}{r+1}}+K_{2}
$$

where $K_{1}$ and $K_{2}$ are arbitrary constants.

Case 4.3 Here for $n=\frac{1-\alpha}{2}$, we find that $\xi_{1}=x^{\frac{1-\alpha}{2}}$ and $\eta_{1}=\eta_{2}=0$ with $A, A_{2}$ as constants. This falls in Case 2.

Case 5. $F, G$ being quadratic functions of $f$ and $g$, i.e., $F_{1}(f, g)=\alpha_{1}\left(f^{2}-g^{2}\right)+\alpha_{2} f+\alpha_{3}$ and $F_{2}(f, g)=2 \alpha_{1} f g+\alpha_{2} g, \alpha_{1} \neq 0$, we have the following four subcases.

Case 5.1 If $n=2 \alpha+3, \alpha_{2}=0$ and $\alpha_{3}=0$, we find that $\xi_{1}=x, \xi_{2}=0, \eta_{1}=-(\alpha+1) f, \eta_{2}=$ $-(\alpha+1) g$ and $A_{1}, A_{2}$ are constants. This case is covered in Case 5.1. 
Case 5.2 By taking $n=2 \alpha+3, \alpha \neq-1, \alpha_{2}^{2}=4 \alpha_{1} \alpha_{3}$, Equation (5) with (7) yields $\xi_{1}=x, \xi_{2}=0, \eta_{1}=-(1+\alpha)\left(f+\frac{\alpha_{2}}{2 \alpha_{1}}\right), \eta_{2}=-(1+\alpha) g$, and $A_{1}=\frac{\alpha_{2} \alpha_{3}}{6 \alpha_{1}} x^{3 \alpha+3}, A_{2}=0$. So two Noether-like operators are found which are of the form

$$
\mathbf{X}_{1}=x \frac{\partial}{\partial x}-(1+\alpha)\left[\left(\frac{\alpha_{2}}{2 \alpha_{1}}+f\right) \frac{\partial}{\partial f}+g \frac{\partial}{\partial g}\right] \text { and } \mathbf{X}_{2}=(1+\alpha)\left[-g \frac{\partial}{\partial f}+\left(\frac{\alpha_{2}}{2 \alpha_{1}}+f\right) \frac{\partial}{\partial g}\right]
$$

Insertion of these operators $\mathbf{X}_{1}$ and $\mathbf{X}_{2}$ in (6) along with pair of real Lagrangians (7), we deduce the following first integrals

$$
\begin{aligned}
& I_{1}=\frac{1}{2} x^{2 \alpha+4}\left(f^{\prime 2}-g^{\prime 2}\right)+\frac{1}{3} \alpha_{1} x^{3 \alpha+3}\left(f^{3}-3 f g^{2}\right)+\frac{1}{2} \alpha_{2} x^{3 \alpha+3}\left(f^{2}-g^{2}\right)+\alpha_{3} x^{3 \alpha+3} f+(\alpha+1) x^{2 \alpha+3} \times \\
& \quad\left(f f^{\prime}-g g^{\prime}\right)+(\alpha+1) \frac{\alpha_{2}}{2 \alpha_{1}} x^{2 \alpha+3} f^{\prime}+\left(\frac{\alpha+1}{3 \alpha+3}\right)\left(\frac{\alpha_{2} \alpha_{3}}{2 \alpha_{1}}\right) x^{3 \alpha+3} \\
& I_{2}=x^{2 \alpha+4} f^{\prime} g^{\prime}+\frac{1}{3} \alpha_{1} x^{3 \alpha+3}\left(3 f^{2} g-g^{3}\right)+\alpha_{2} x^{3 \alpha+3}(f g)+\alpha_{3} x^{3 \alpha+3} g+(\alpha+1) x^{2 \alpha+3}\left(f g^{\prime}+f^{\prime} g\right)+ \\
& \quad(\alpha+1) \frac{\alpha_{2}}{2 \alpha_{1}} x^{2 \alpha+3} g^{\prime} .
\end{aligned}
$$

On closer view it is found that the operator $\mathbf{X}_{1}$ is also a Noether symmetry for the LE-system (2) and the classical Noether theorem along with (7) produces the first integrals identical to (13). From the commutation relations, it can be checked that the operators $\mathbf{X}_{1}$ and $\mathbf{X}_{2}$ form an Abelian algebra, i.e., $\left[\mathbf{X}_{1}, \mathbf{X}_{2}\right]=0$.

Moreover, by introducing $w_{1}=x^{1+\alpha} f+\frac{\alpha_{2}}{2 \alpha_{1}} x^{\alpha+1}$ and $w_{1}=x^{\alpha+1} g$, where $w=f+i g$, Equation (13) can be transformed into the following first-order variable separable form

$$
C=\frac{1}{2}(\alpha+1)^{2} w^{2}-\frac{1}{2} x^{2} w^{\prime 2}-\frac{1}{3} \alpha_{1} w^{3} .
$$

Case $5.3 n=\frac{\alpha+4}{3}, n \neq \frac{1-\alpha}{2},-1, \alpha_{2}=0$ and $\alpha_{3}=0$, we get $\xi_{1}=x^{\frac{2-\alpha}{3}}, \eta_{1}=-\frac{\alpha+1}{3} x^{-\frac{\alpha+1}{3}} f$, $\eta_{2}=-\frac{\alpha+1}{3} x^{-\frac{\alpha+1}{3}} g$ and $A_{1}=\frac{(\alpha+1)^{2}}{18}\left(f^{2}-g^{2}\right)+c, A_{2}=\frac{(\alpha+1)^{2}}{9} f g$, where $c$ is a constant. This case can be absorbed in Case $\mathbf{5 . 2}$ shown below.

Case 5.4 If $n=\frac{1-\alpha}{2}$ with $n_{1} \neq \frac{\alpha+4}{3}$ and $\alpha_{2}, \alpha_{3}$ are arbitrary constants, we find $\xi_{1}=x^{\frac{1-\alpha}{2}}, \xi_{2}=$ $0, \& \eta_{1}, \eta_{2}=0$. This subcase is contained in Case 2.

Case 6. For the choices $F(f, g)=\alpha_{1} \exp \left(\alpha_{2} f\right) \cos \left(\alpha_{2} g\right)+\alpha_{3} f+\delta$ and $G(f, g)=$ $\alpha_{1} \exp \left(\alpha_{2} f\right) \sin \left(\alpha_{2} g\right)+\alpha_{3} g$, where $\alpha_{1} \neq 0, \alpha_{2} \neq 0$, we consider two subcases here.

Case 6.1 If $n=\frac{1-\alpha}{2}$, Equation (5) gives $\xi_{1}=x^{\frac{1-\alpha}{2}}$ with $\eta_{1}=\eta_{2}=0$ and $A_{1}=A_{2}=k, k$ a constant. This reduces to Case 2.

Case 6.2 If $n=1, \alpha \neq-1, \alpha_{3}=0$ and $\delta=0$, from the Noether-like symmetry condition (5) on using (7) we determine that $\xi_{1}=x, \xi_{2}=0$ and $\eta_{1}=-\frac{\alpha+1}{\alpha_{2}}, \eta_{2}=0$ with $A_{1}=A_{2}=k, k$ a constant. Therefore we have two Noether-like operators, $\mathbf{X}_{1}=x \frac{\partial}{\partial x}-\frac{\alpha+1}{\alpha_{2}} \frac{\partial}{\partial f}, \mathbf{X}_{2}=\frac{\alpha+1}{\alpha_{2}} \frac{\partial}{\partial g}$. Utilization of these operators $\mathbf{X}_{1}$ and $\mathbf{X}_{2}$ along with the particular pair of real Lagrangians (7) in Equation (6) provides the two first integrals

$$
\begin{aligned}
& I_{1}=\frac{1}{2} x^{2}\left(f^{\prime 2}-g^{\prime 2}\right)+\frac{1}{\alpha_{2}} x^{1+\alpha} \exp \left(\alpha_{2} f\right) \cos \left(\alpha_{2} g\right)+\frac{(\alpha+1)}{\alpha_{2}} x f^{\prime} \\
& I_{2}=x^{2} f^{\prime} g^{\prime}+\frac{1}{\alpha_{2}} x^{1+\alpha} \exp \left(\alpha_{2} f\right) \sin \left(\alpha_{2} g\right)+\frac{(\alpha+1)}{\alpha_{2}} x g^{\prime} .
\end{aligned}
$$

One can check that the Noether-like operators $\mathbf{X}$ in this case is a Noether symmetry of the corresponding Euler-Lagrange equation (2) and the standard Noether's theorem provides the same first integrals as given in (15) while the associated Lagrangians $L_{1}$ and $L_{2}$ are found in (7). Use of transformation $u=\frac{\alpha+1}{\alpha_{2}} \ln (w / x)$ can easily express (15) in an integrable form 


$$
\int \frac{d w}{ \pm \sqrt{1-2 \alpha_{1} \alpha_{2}(\alpha+1)^{-2} w^{\alpha+1}+2 K_{1} \alpha_{2}^{2}(\alpha+1)^{-2}}}=\ln x K_{2}
$$

where $K_{1}$ and $K_{2}$ are integration constants. We find that Lie algebra of these operators is Abelian, i.e., $\left[\mathbf{X}_{1}, \mathbf{X}_{2}\right]=0$.

Case 7 For $n \neq \frac{1-\alpha}{2}$, and $F$ and $G$ are arbitrary but not of the forms presented in the cases, $\mathbf{1}, \mathbf{3}, \mathbf{4}, \mathbf{5}, \mathbf{6}$, the pair of equations (5) yields $\xi_{1}=\xi_{2}=0, \eta_{1}=\eta_{2}=0$ with $A_{1}=A_{2}=$ constant, so in this case no Noether-like operator exists and we cannot find first integrals of the underlying system of Euler-Lagrange equations.

$$
\text { B. } P(X)=\beta X^{-2 n}, n \neq 1, \beta \text { a Constant }
$$

By assuming this specific form of $P(x)$ in (2) and accordingly in (7), we find that:

Case 1. For $F=\exp \left(\beta_{1} f\right) \cos \left(\beta_{1} g\right)$ and $G=\exp \left(\beta_{1} f\right) \sin \left(\beta_{1} g\right)$, Equation (5) with the associated Lagrangians provides a single Noether-like operator $\mathbf{X}=x^{n} \frac{\partial}{\partial x}$ with gauge terms $A_{1}=A_{2}=0$. So with this operator $\mathbf{X}$, and invocation of (6) with the aid of (7) gives rise to the following conserved quantities

$$
\begin{aligned}
& I_{1}=\frac{1}{2} x^{2 n}\left(f^{\prime 2}-g^{\prime 2}\right)-\frac{\beta}{\beta_{1}} \exp \left(\beta_{1} f\right) \cos \left(\beta_{1} g\right) \\
& I_{2}=x^{2 n} f^{\prime} g^{\prime}-\frac{\beta}{\beta_{1}} \exp \left(\beta_{1} f\right) \sin \left(\beta_{1} g\right)
\end{aligned}
$$

for the system (2). Moreover, we find that the operator $\mathbf{X}$ is also a Noether symmetry generator of the variational system (2). Hence the application of the classical Noether's theorem with Lagrangians (7) yields the two first integrals which are identical to (17). Furthermore, (17) can be cast in an integrable form as

$$
\int \frac{d u}{ \pm \sqrt{2 C_{1}-2 \exp \left(\beta_{1} u\right) / \beta_{1}}}=\frac{x^{1-n}}{1-n}+C_{2}
$$

where $u=f+i g$.

As a consequence of the above two cases 3.1 and 3.2 we can summarize all the independent cases in which Noether-like operators and related first integrals exist in the Table 1:

Table 1. Noether-like operators and first integrals.

\begin{tabular}{lll}
\hline Cases & Noether-Like Operators & First Integrals \\
\hline Case A-2 & $\mathbf{X}=x^{\frac{1-\alpha}{2}} \frac{\partial}{\partial x}$ & $I_{1}=\frac{1}{2} x^{1-\alpha}\left(f^{\prime 2}-g^{\prime 2}\right)+\int(F d f-G d g)$ \\
$I_{2}=x^{1-\alpha}\left(f^{\prime} g^{\prime}\right)+\int(G d f+F d g)$
\end{tabular}


Table 1. Cont.

\begin{tabular}{lll}
\hline Cases & Noether-Like Operators & First Integrals \\
\hline \multirow{2}{*}{ Case A-6.2 } & $\mathbf{X}_{1}=x \frac{\partial}{\partial x}-\frac{\alpha+1}{\alpha_{2}} \frac{\partial}{\partial f}$ & $I_{1}=\frac{1}{2} x^{2}\left(f^{2}-g^{2}\right)+\frac{1}{\alpha_{2}} x^{1+\alpha} \exp \left(\alpha_{2} f\right) \cos \left(\alpha_{2} g\right)+\frac{(\alpha+1)}{\alpha_{2}} x f^{\prime}$ \\
& $\mathbf{X}_{2}=\frac{\alpha+1}{\alpha_{2}} \frac{\partial}{\partial g}$ & $I_{2}=x^{2} f^{\prime} g^{\prime}+\frac{1}{\alpha_{2}} x^{1+\alpha} \exp \left(\alpha_{2} f\right) \sin \left(\alpha_{2} g\right)+\frac{(\alpha+1)}{\alpha_{2}} x g^{\prime}$ \\
\hline \multirow{2}{*}{ Case B-1 } & $\mathbf{X}=x^{n} \frac{\partial}{\partial x}$ & $I_{1}=\frac{1}{2} x^{2 n}\left(f^{\prime 2}-g^{\prime 2}\right)-\frac{\beta}{\beta_{1}} \exp \left(\beta_{1} f\right) \cos \left(\beta_{1} g\right)$ \\
& & $I_{2}=x^{2 n} f^{\prime} g^{\prime}-\frac{\beta}{\beta_{1}} \exp \left(\beta_{1} f\right) \sin \left(\beta_{1} g\right)$ \\
\hline
\end{tabular}

\section{Conclusions}

Though ample literature on the discussion of Noether symmetries and corresponding first integrals for scalar ODEs is available, there is still paucity of work done on operators and associated conserved quantities of two-dimensional systems of nonlinear ODEs. In this work we have used the complex Lagrangian formulation to study integral properties of a wide class of dynamical problems which are expressible as a two-dimensional LE-system. We have performed the classification of a generalized class of LE-systems with respect to Noether-like operators and established first integrals. To the best of our knowledge this classification has not been performed so far and we have investigated eight classes of the generalized version of LE-system. Amongst the eight classes only eight are found to be independent and the algebra of Noether-like operators is Abelian in few cases. Moreover, we have seen that the five cases amongst the seven are those which possess the same first integrals if either we utilize the first-order Lagrangians in the Noether-like theorem or as in the classical Noether's theorem. We also point out here that in few cases the pairs of first integrals can also be transformed via quadrature. To conclude our discussion we mention that for coupled systems of two nonlinear LE-equations, the construction of Lagrangian will of course be a highly difficult task. However, we have seen here that the complex Lagrangian approach can remarkably minimize the complexity of the problem and nontrivial results can be achieved for a wide class of Euler-Lagrange systems as we have seen in the LE-system. Lastly, we have discussed the generalized LE-system by introducing various forms of $F(f, g)$ and $G(f, g)$ and by considering only two forms of $P(x)$. Thus, in future work if one wants to study the role of $P(x)$ with multiple forms, this analysis may be helpful to explore even more properties of LE-systems and their applications.

Author Contributions: Problem investigation, methodology, M.U.F.; formal analysis, F.M.M.; supervision, C.M.K. Funding: This research received no external funding.

Acknowledgments: F.M. thanks the N.R.F. of South Africa for support.

Conflicts of Interest: The authors declare no conflict of interest.

\section{References}

1. Chandrasekar, S. An Introduction to the Study of Stellar Structure; Dover: New York, NY, USA, 1957.

2. Thomson, W. Collected Papers; Cambridge University Press: Cambridge, UK,1991; Volume 5, p. 266.

3. Emden, R. Gaskugein: Anwendungen der Mechanischen Warmen-Theories auf Kosmologei und Meteorologische Probleme; Teubner: Leipzig, Germany, 1907.

4. Fowler, R.H. The form near infinity of real continuous solutions of a certain differential equation of the second order. Quar. J. Math. 1914, 45, 289-350.

5. Fowler, R.H. Further studies of Emden's and similar differential equations. Quar. J. Math. 1931, 2, $259-288$. [CrossRef]

6. Richardson, O.W. The Emission of Electricity from Hot Bodies, 2nd ed.; Longmans, Green and Company: London, UK, 1921.

7. Dehghan, M.; Shakeri, F. Approximate solution of a differential equation arising in astrophysics using the variational iteration method. New Astron. 2008, 13, 53-59. [CrossRef]

8. Ramos, I.J. Series approach to the Lane-Emden equation and comparison with the homotopy perturbation method. Chaos Solit. Fract. 2008, 38, 400-408. [CrossRef] 
9. Marzban,H.R.; Tabrizidooz, H.R.; Razzaghi, M. Hybrid functions for nonlinear initial value problems with applications to Lane-Emden type equations. Phys. Lett. A 2008, 372, 5883-5886. [CrossRef]

10. Wong, J.S.W. On the generalized Emden-Fowler equation. SIAM Rev. 1975, 17, 339-360. [CrossRef]

11. Khalique, C.M.; Mahomed, F.M.; Muatjetjeja, B. Lagrangian formulation of generalized Lane-Emden equation and double reduction. J. Nonlinear Math. Phys. 2008, 15, 152-161. [CrossRef]

12. Khalique, C.M.; Muatjetjeja, B. Group classification of the generalized Lane-Emden type equation. Nonlinear Anal. Real World Appl. 2009, 10, 3387-3395. [CrossRef]

13. Zou, H. A priori estimates for a similar elliptic system without variational structure and their applications. Math. Ann. 2002 323, 713-735. [CrossRef]

14. Serrin, J.; Zou, H. Non-existence of positive solutions of Lane-Emden systems. Differ. Int. Equ. 1996, 9,635-653.

15. Serrin, J.; Zou, H. Existence of positive solutions of the Lane-Emden system. Atti del Seminario Matematico e Fisico Dell Universit'a di Modena 1998, 46, 369-380.

16. Qi, Y.W. The existence of ground states to a weakly coupled elliptic system. Nonlinear Anal. Theo. Meth. Appl. 2002, 48, 905-925. [CrossRef]

17. Dalmasso, R. Existence and uniqueness of solutions for a semilinear elliptic system. Int. J. Math. Math. Sci. 2005, 10, 1507-1523. [CrossRef]

18. Farooq, M.U. Noether-Like operators and first integrals for generalized systems of Lane-Emden equations. Symmetry 2019, 11, 162. [CrossRef]

19. Muatjetjeja, B.; Khalique, C.M. Noether, partial Noether operators and first integrals for the coupled Lane-Emden system. Math. Comput. Appl. 2010, 15, 325-333. [CrossRef]

20. Muatjetjeja, B.; Khalique, C.M. First integrals for generalized coupled Lane-Emden system. Nonlinear Anal. Real World Appl. 2011, 12, 1202-1212. [CrossRef]

21. Muatjetjeja, B.; Khalique, C.M. Lagrangian approach to a generalized coupled Lane-Emden system: Symmetries and first integrals. Commu. Nonlinear Sci. Numer. Simul. 2010, 15, 1166-1171. [CrossRef]

22. Ali, S.; Mahomed, F.M.; Qadir, A. Complex Lie symmetries for variational problems. J. Nonl. Math. Phys. 2008, 15, 2535. [CrossRef]

23. Farooq, M.U.; Ali, S.; Qadir, A. Invariants of two-dimensional systems via complex Lagrangians with applications. Commun. Nonl. Sci. Num. Simul. 2011, 16, 1804-1810. [CrossRef]

24. Farooq, M.U.; Ali, S.; Mahomed, F.M. Two dimensional systems that arise from the Noether classification of Lagrangian on the line. Appl. Math. Comput. 2011, 217, 6959-6973. [CrossRef]

25. Ali, S.; Mahomed, F.M.; Qadir, A. Linearizability criteria for systems of two second-order differential equations by complex methods. Nonlinear Dyn. 2011, 66, 77-88. [CrossRef]

26. Mahomed, F.M.; Qadir, A.; Ramnarain, A. Laplace-Type Semi-Invariants for a System of Two Linear Hyperbolic Equations by Complex Methods. Math. Probl. Eng. 2011, 2011, 202973. [CrossRef]

27. Naz, R.; Mahomed, F.M. Lie and Noether symmetries of systems of complex ordinary differential equations and their split systems. Pramana J. Phys. 2014, 83, 920. [CrossRef]

28. Qadir, A.; Mahomed, F.M. Higher dimensional systems of differential equations obtainable by iterative use of complex methods. Int. J. Mod. Phys. 2015, 38, 1560077. [CrossRef]

29. Noether, E. Invariante Variations probleme. Nachrichten von der Gesellschaft der Wissenschaften zu Göttingen, Mathematisch-Physikalische Klasse 1918, 2, 235-296.

(C) 2019 by the authors. Licensee MDPI, Basel, Switzerland. This article is an open access article distributed under the terms and conditions of the Creative Commons Attribution (CC BY) license (http://creativecommons.org/licenses/by/4.0/). 